\title{
SENTINEL LYMPH NODE BIOPSY AFTER NEOADJUVANT CHEMOTHERAPY IN WOMEN WITH BREAST CANCER: CLINICAL PROFILE AND PROGNOSIS
}

Marcelo Bello1, Anke Bergmann"1, Marcelo Barbosa, Suzana Aguiar ${ }^{1}$, Fabiana Tonellotto1, Sergio Monteiro1, Patricia Juca', Luiz Claudio Santos Thuler ${ }^{1}$

${ }^{1}$ National Cancer Institute - Rio de Janeiro (RJ), Brazil.

Objectives: Describe the frequency and factors associated with the indication of Sentinel lymph node biopsy (SLNB) after neoadjuvant chemotherapy (NACT) and the impact of SLNB on prognosis. Methods: Retrospective cohort study of women with breast cancer submitted to NACT from january 2013 to july 2015 at Hospital do Cancer III of the National Cancer Institute (HCIII/INCA). The data were collected in electronic and physical records. This study was approved by INCA's ethics and research committee. Results: A total of 783 women with mean age of 52 years $( \pm 11)$ were included. The majority were diagnosed with clinical stage IIIA (23\%) and IIIB (33\%), and with a Luminal A subtype (ER+ and / or PR+, HER2-) (52\%). After NACT, complete response was observed in 15\% and partial in 58\%. Mastectomy was performed in $85 \%$ of the women, and 25\% were submitted to SLNB (alone 14\% and SLNB + Lymphadenectomy 11\%). The factors associated with SLNB were: Initial clinical stage (OR $=6.22$ 95\%CI 4.28-9.06) and complete response to NACT (OR=1.96 95\%CI 1.29-2.97). In the follow-up, were observed $23 \%$ of recurrence /metastasis and $13 \%$ of death. After adjustment (cT, cN and NACT response), there was no association between the performance of SLNB with recurrence/metastasis (HR=1.51 95\%CI 0.93-2.46) and death (HR=1.48 95\%CI 0.76-2.90). Conclusion: The indication of SLNB after NACT was associated with initial clinical stage and complete response to NACT. After adjustment, SLNB was not associated with prognosis. 